\title{
ON THE ZEROS OF HILBERT SPACES OF ANALYTIC FUNCTIONS
}

\author{
S. H. KON
}

(Received 9 June 1975; revised 9 September 1975)

\begin{abstract}
An attempt is made to characterise the zeros of some Hilbert spaces of analytic functions means of their kernel functions. Results on the zeros of functions in $D_{\phi}$ and their uniqueness $s$ are included, in particular we give an affirmative answer to a question of Shapiro and Shield
\end{abstract}

\section{Introduction}

Let $U$ be the open unit disc and $H(U)$ the set of all analytic functions in Let $\phi(z)=\Sigma c_{n} z^{n} \in H(U)$, with $c_{0}=1, c_{n}>0$ and

$$
c_{n}^{2} \leqq c_{n-1} c_{n+1} .
$$

For $f(z)=\Sigma a_{n} z^{n} \in H(U)$, define

$$
\|f\|^{2}=\Sigma \frac{1}{c_{n}}\left|a_{n}\right|^{2}
$$

and let $D_{\phi}=\left\{f \in H(U):\|f\|^{2}<\infty\right\}$. Then $D_{\phi}$ is a Hilbert space under the inn product

$$
(f, g)=\Sigma \frac{1}{c_{n}} a_{n} \bar{b}_{n}
$$

where $g(z)=\Sigma b_{n} z^{n}$.

Let

$$
D=\left\{f \in H(U): f(0)=0, \quad \frac{1}{\pi} \iint_{U}\left|f^{\prime}(z)\right|^{2} d x d y=\Sigma n\left|a_{n}\right|^{2}<\infty\right\} .
$$

Then $D$ is also a Hilbert space under the inner product $(f, g)=\sum n a_{n} \bar{b}_{n}$.

The reproducing kernel for $D_{\phi}$ is $K_{\xi}(z)=\phi\left(\bar{\xi}_{z}\right), \xi \in U$. That is, for a $\xi \in U$,

$$
f(\xi)=\left(f, K_{\xi}\right) \quad \text { for } \quad \text { all } \quad f \in D_{\phi} .
$$


The reproducing kernel for $D$ is $K_{\xi}(z)=-\log \left(1-\bar{\xi}_{z}\right)$. For simplicity we write $K_{n}$ for $K_{\xi}$ when $\xi=z_{n}$.

A set $\left\{z_{n}\right\}$ in $U$ is called a set of uniqueness for a subspace $\mathscr{F}$ of $H(U)$ if $f \in \mathscr{F}$ and $f\left(z_{n}\right)=0$ for all $n$ imply $f \equiv 0$.

The following two results are due to Shapiro \& Shields (1962).

THEOREM 1. If $\left\{z_{n}\right\}$ is any sequence of points in $U$ for which

$$
\sum \frac{1}{K_{n}\left(z_{n}\right)}<\infty
$$

then there is an $f(\not \equiv) \in D_{\phi}$ vanishing at all these points (with a similar result for $D$ ).

THEOREM 2. Let $h(t)$ be any continuous function with $h(0)=0, h(t)>0$ $(t>0)$. Then there exists a set of uniqueness $\left\{z_{n}\right\}$ for $D$ satisfying the condition

$$
\sum \frac{1}{-\log \left(1-\left|z_{n}\right|\right)} h\left(1-\left|z_{n}\right|\right)<\infty .
$$

Shapiro \& Shields (1962, page 224) observed that Theorem 2 holds for $D_{\phi}$ when $\phi(z)=(1-z)^{\alpha-1}$ for $0 \leqq \alpha<1$. They raised the question whether this is true for all $D_{\phi}$. We give this an affirmative answer in section 2. Examples of $D_{\phi}$ with zero sets violating (2) are also given.

\section{Uniqueness sets and zeros of functions in $D_{\phi}$}

If (1) holds and $\phi(z) \in H(U)$ then as shown by Shapiro and Shields (1962, Lemma 6),

$$
1=c_{0} \geqq c_{1} \geqq c_{2} \geqq \cdots .
$$

The first lemma gives an estimate on the norm of a function with specified zeros.

Lemma 1. Let $z_{1}, z_{2}, \cdots z_{n}$ be $n$ equally spaced points on the circle $|z|=r$, $0<r<1$. If $f \in D_{\phi}$ with $f\left(z_{i}\right)=0(i \leqq n)$ and $f(0)=1$ then

$$
\|f\|^{2} \geqq n / \phi\left(r^{2}\right) \text {. }
$$

Proof. Without loss of generality take $z_{1}=r$. Define $h(z)$ by $h=$ $1 / n\left(K_{1}+K_{2}+\cdots+K_{n}\right)$. Then $(f, h)=0$ and so

$$
1=(f, 1)=(f, 1-h) \leqq\|f\|\|1-h\| \text {. }
$$

Further

$$
\|1-h\|^{2}=\sum_{m=1}^{\infty} c_{n m} r^{2 n m} \leqq \frac{1}{n} \phi\left(r^{2}\right)
$$

since $c_{k}$ and $r^{2 k}$ decreases as $k$ increases. 
LEMMA 2. For any sequence $r_{k}, 0<r_{k}<1$ there is a sequence of integers $n$ such that

$$
\frac{2}{k}>\frac{\phi\left(r_{k}^{2}\right)}{n_{k}} \geqq \frac{1}{k}
$$

Proof. Let $N_{k}=\left\{n: \phi\left(r_{k}^{2}\right) \geqq n / k, n\right.$ integer $\}$. Since $\phi\left(r_{k}^{2}\right)>1$, the set $N_{k}$ i: non-empty and is obviously bounded. Let $n_{k}=\max N_{k}$, then

$$
\frac{\phi\left(r_{k}^{2}\right)}{n_{k}} \geqq \frac{1}{k}>\frac{\phi\left(r_{k}^{2}\right)}{n_{k}+1} \geqq \frac{\phi\left(r_{k}^{2}\right)}{2 n_{k}} .
$$

CorollaRY. If $h(t)$ is continuous and $h(0)=0, h(t)>0$, then there is a sequence $r_{n}, 0<r_{n}<1$ such that

$$
\sum \frac{1}{\phi\left(r_{n}^{2}\right)}=\infty \quad \text { while } \quad \sum \frac{1}{\phi\left(r_{n}^{2}\right)} \cdot h\left(1-r_{n}\right)<\infty .
$$

Proof. For each $k$, choose $s_{k}, 0<s_{k}<1$ such that $h\left(1-s_{k}\right)<\frac{1}{k^{3}}$. For this sequence choose a sequence of integers $\left\{n_{k}\right\}$ as in Lemma 2 . Let $\left\{r_{n}\right\}$ be the sequence obtained by repeating $n_{k}$ times each $s_{k}$.

We can now prove the following:

THeOREM 3. Let $h(t)$ be any continuous function with $h(0)=0, h(t)>($ $(t>0)$. Then there exists a set of uniqueness $\left\{z_{n}\right\}$ for $D_{\phi}$ satisfying the condition

$$
\sum \frac{1}{K_{n}\left(z_{n}\right)} \cdot h\left(1-\left|z_{n}\right|\right) \equiv \sum \frac{1}{\phi\left(\left|z_{n}\right|^{2}\right)} \cdot h\left(1-\left|z_{n}\right|\right)<\infty .
$$

Proof. Choose $r_{k}, 0<r_{k}<1$ such that $h\left(1-r_{k}\right)<1 / k^{3}$ and then $n_{k}$ as ir Lemma 2. Now set

$$
\left\{z_{n}\right\}=\bigcup_{k=1}^{\infty}\left\{z=r_{k} e^{2 \pi m i / n_{k}}: m=0,1, \cdots, n_{k}-1\right\}
$$

Then

$$
\sum_{n} \frac{h\left(1-\left|z_{n}\right|\right)}{\phi\left(\left|z_{n}\right|^{2}\right)}=\sum_{k} \frac{n_{k} h\left(1-r_{k}\right)}{\phi\left(r_{k}^{2}\right)} \leqq \sum_{k} \frac{1}{k^{2}}<\infty .
$$

If $f(\not \equiv 0) \in D_{\phi}$ with $f\left(z_{n}\right)=0$, for all $n$, we can suppose for our purpose that $f(0)=1$. Then by Lemma 1

$$
\|f\|^{2} \leqq n_{k} / \phi\left(r_{k}^{2}\right)>k / 2, \quad k=1,2,3, \cdots .
$$

Hence $\left\{z_{n}\right\}$ is a uniqueness set for $D_{\phi}$.

For $\phi(z)=\Sigma z^{n}, D_{\phi}$ is just merely the Hardy space $H^{2}$ and $1 / K_{n}\left(z_{n}\right)<\infty$ is then equivalent to the Blaschke condition which is necessary and sufficient for $\left\{z_{n}\right\}$ to be a zero set of $H^{2}$. Apart from this case, it is easy to show that for $\phi(z)$ 
with radius of convergence $R>1, D_{\phi} \subseteq H(\bar{U})$ the class of functions analytic in a neighbourhood of the closed unit disc $\bar{U}$. Obviously here the non-trivial zero sets are just the finite subsets of $U$. As against that we have the following situation.

THEOREM 4. If the Taylor coefficients $c_{n}$ of $\phi$ satisfy $1 / c_{n}=0\left(n^{k}\right)$ for some positive integer $k$ and $c_{n} \rightarrow 0$, then there exists an $f(\not \equiv 0) \in D_{\phi}$, whose zeros $\left\{z_{n}\right\}$ satisfy

$$
\sum \frac{1}{K_{n}\left(z_{n}\right)}=\infty
$$

Proof. The function $h(t)=t \phi\left((1-t)^{2}\right) \rightarrow 0$ as $t \rightarrow 0$. To see this observe that $N$ can be chosen for each $n$ such that $n c_{k}<\frac{1}{2}$ for $k \geqq N$. Then

$$
n \phi\left(r^{2}\right) \leqq n\left(c_{0}+\cdots+c_{N-1} r^{2(N-1)}\right)+\frac{1}{2} r^{2 N} \cdot \frac{1}{1-r^{2}} \leqq \frac{1}{1-r^{2}}
$$

for $r$ close to 1 . The Corollary to Lemma 2 now applies to give $\left\{z_{n}\right\}$ on the unit interval such that

$$
\sum\left(1-z_{n}\right)<\infty \quad \text { and } \quad \sum \frac{1}{\phi\left(z_{n}^{2}\right)}=\infty .
$$

By a result of Caughran (1969, Theorem 2) there exists an $f(\not \equiv 0) \in H(U)$ with bounded $f^{(k)}$ and vanishing at all the points $z_{n}$. Note that

$$
\sum_{n} n^{k}\left|a_{n}\right|<\infty \quad \text { if } \quad \iint_{U}\left|f^{(k)}(z)\right|^{2} d x d y<\infty
$$

and since $1 / c_{n}=0\left(n^{k}\right)$, this implies that $f \in D_{\phi}$.

\section{References}

J. G. Caughran (1969), 'Two results concerning the zeros of functions with finite Dirichlet integrals', Canad. J. Math. 21, 312-316.

H. S. Shapiro and A. L. Shields (1962), 'On the zeros of functions with finite Dirichlet integral and some related function spaces', Math.' $Z$. 80, 217-229.

Department of Mathematics, University of Malaya, Kuala Lumpur 22-11, Malaysia. 